\title{
Derived Categories of Toric Varieties
}

\author{
Yujiro Kawamata
}

October 15, 2018

\section{Introduction}

The purpose of this paper is to investigate the structure of the derived category of a toric variety. We shall prove the following:

Theorem 1.1. Let $X$ be a projective toric variety with at most quotient singularities, let $B$ be an invariant $\mathbb{Q}$-divisor whose coefficients belong to the set $\left\{\frac{r-1}{r} ; r \in \mathbb{Z}_{>0}\right\}$, and let $\mathcal{X}$ be the smooth Deligne-Mumford stack associated to the pair $(X, B)$ as in [12]. Then the bounded derived category of coherent sheaves $D^{b}($ Coh $(\mathcal{X}))$ has a complete exceptional collection consisting of sheaves.

An object of a triangulated category $a \in T$ is called exceptional if

$$
\operatorname{Hom}^{p}(e, e) \cong \begin{cases}\mathbb{C} & \text { for } p=0 \\ 0 & \text { for } p \neq 0\end{cases}
$$

A sequence of exceptional objects $\left\{e_{1}, \ldots, e_{m}\right\}$ is said to be an exceptional collection if

$$
\operatorname{Hom}^{p}\left(e_{i}, e_{j}\right)=0 \text { for all } p \text { and } i>j .
$$

It is said to be strong if in addition that $\operatorname{Hom}^{p}\left(e_{i}, e_{j}\right)=0$ for $p \neq 0$ and all $i, j$. It is called complete if $T$ coincides with the smallest triangulated subcategory containing all the $e_{i}$ (cf. [2]).

It is usually hard to determine the explicit structure of a derived category of a variety. But it is known that some special varieties such as a projective space or a Grassmann variety have strong complete exceptional collections consisting of vector bundles ([1], [8, [9, [10]). Such sheaves are 
useful for further investigation of the derived categories ([18, [7], [14] and [6] for example).

We use the minimal model program for toric varieties as developed in [17] (and corrected in [15]) in order to prove the theorem. A special feature for this approach is that, even if we start with the smooth and non-boundary case $B=0$, we are forced to deal with not only singularities but also the case $B \neq 0$ because Mori fiber spaces have multiple fibers in general. Thus we are inevitably lead to consider the general situation concerning Deligne-Mumford stacks even if we only need results for smooth varieties. The stacky sheaves need careful treatment because there exist non-trivial stabilizer groups on the stacks (cf. Remark 5.1),

We start with the Beilinson theorem for the case of projective spaces, and build up exceptional collections following the procedure of the minimal model program. We use a covering trick to proceed from projective spaces to log Fano varieties (§3). Then we proceed by induction on the dimension. First we consider a Mori fiber space in $\S 4$ where the base space is assumed to have already a complete exceptional collection by the induction hypothesis. Though a Mori fiber space has singular fibers, the associated morphism of stacks is proved to be smooth (Corollary 4.2), and we can define a complete exceptional collection on the total space by using twisted pull-backs. The behavior of derived catogories under the birational transformations such as a divisorial contraction or a flip was studied in 12 . We use this result together with results of $\S 4$ in $\S 5$ and $\S 6$. Indeed, the exceptional locus of a divisorial contraction or a flip has a structure of a Mori fiber space itself. The argument of the proof is a generalization of that in [16] which considered the derived categories of a projective space bundle and a blowings-up with a smooth center in a smooth variety.

\section{Toric minimal model program}

Let $X$ be a projective toric variety of dimension $n$ which is quasi-smooth, i.e., has only quotient singularities. We note that a toric variety is quasi-smooth if and only if it is $\mathbb{Q}$-factorial. We consider a $\mathbb{Q}$-divisor $B$ on $X$ whose prime components are invariant divisors with coefficients being contained in the set $\left\{\frac{r-1}{r} ; r \in \mathbb{Z}_{>0}\right\}$. Let $\mathcal{X}$ be the smooth Deligne-Mumford stack associated to the pair $(X, B)$ with the natural morphism $\pi_{X}: \mathcal{X} \rightarrow X$ as in [12].

The pair $(X, B)$ has only log terminal singularities. We work on the 
log minimal model program for $(X, B)$. We refer the reader to [17] or [15]. Let $\phi: X \rightarrow Y$ be a primitive contraction morphism corresponding to an extremal ray with respect to $K_{X}+B$. Then $Y$ is also a projective toric variety and $\phi$ is a toric morphism. If $\phi$ is a birational morphism, then the boundary divisor $C$ on $Y$ is defined to be the strict transform of $B$. Otherwise, it will be defined later.

Let $N_{X}$ be the lattice of 1-parameter subgroups of the torus acting on $X$, and $\Delta_{X}$ the fan in $N_{X, \mathbb{R}}$ corresponding to $X$. Let $w=\left\langle v_{3}, \ldots, v_{n+1}\right\rangle$ be a wall in $\Delta_{X}$ corresponding to an extremal rational curve, where the $v_{i}$ are primitive vectors in $N_{X}$ on the edges of $w$. Let $v_{1}$ and $v_{2}$ be two primitive vectors in $N_{X}$ each of which forms an $n$-dimensional cone in $\Delta_{X}$ when combined with $w$. Let $D_{i}$ be the prime divisors on $X$ corresponding to the $v_{i}$, and $\mathcal{D}_{i}$ the corresponding prime divisors on $\mathcal{X}$. Let $\frac{r_{i}-1}{r_{i}}$ be the coefficients of the $D_{i}$ in $B$. Then the natural morphism $\pi_{X}: \mathcal{X} \rightarrow X$ ramifies along $D_{i}$ such that $\pi_{X}^{*} D_{i}=r_{i} \mathcal{D}_{i}$.

The contraction morphism is described by an equation

$$
a_{1} v_{1}+\cdots+a_{n+1} v_{n+1}=0
$$

where the $a_{i}$ are integers such that

$$
\begin{aligned}
& \left(a_{1}, \ldots, a_{n+1}\right)=1 \\
& a_{i}>0 \text { for } 1 \leq i \leq \alpha \\
& a_{i}=0 \text { for } \alpha+1 \leq i \leq \beta \\
& a_{i}<0 \text { for } \beta+1 \leq i \leq n+1 \\
& 2 \leq \alpha \leq \beta \leq n+1 .
\end{aligned}
$$

Note that we use slightly different notation from the literatures where the $a_{i}$ are rational numbers. Since $K_{X}+B$ is negative for $\phi$, we have

$$
\sum_{i=1}^{n+1} \frac{a_{i}}{r_{i}}>0 .
$$

The following lemma asserts that the set of integers $\left\{a_{i}\right\}$ is well prepared:

Lemma 2.1. Let $i_{0}$ be an integer such that $1 \leq i_{0} \leq \alpha$ or $\beta+1 \leq i_{0} \leq n+1$. Then the set of $n+\alpha-\beta$ integers $a_{i}$ for $1 \leq i \leq \alpha$ and $\beta+1 \leq i \leq n+1$ except one $i=i_{0}$ is coprime for any $i_{0}$. 
Proof. Let $c$ be the largest common divisor of these integers, and set $a_{i}=c \bar{a}_{i}$. Then we have $\left(a_{i_{0}}, c\right)=1$. Let $x, y$ be integers such that $a_{i_{0}} x+c y=1$. Then

$$
\frac{1}{c} v_{i_{0}}=\frac{1}{c} v_{i_{0}}-\frac{x}{c} \sum_{i} a_{i} v_{i}=-x \sum_{i \neq i_{0}} \bar{a}_{i} v_{i}+y v_{i_{0}} \in N_{X}
$$

hence $c=1$.

One of the following cases occurs.

(1) Mori fiber space: $\beta=n+1$. We have $\operatorname{dim} Y=n+1-\alpha$.

(2) divisorial contraction: $\beta=n$.

(3) small contraction: $\beta<n$.

We treat these cases separately in the following sections. According to the minimal model program, Theorem 1.1 follows from the combination of Corollaries 4.4, 5.3 and 6.2

\section{Fano case}

We start with the case where $(X, B)$ is a log $\mathbb{Q}$-Fano variety with $\rho=1$. We have $\alpha=\beta=n+1$. In this case, there are no edges in $\Delta_{X}$ besides $\mathbb{R}_{\geq 0} v_{i}$. Such a variety $X$ is not necessarily a weighted projective space as remarked in [15]. But it is covered by a weighted projective space by a finite morphism which is etale in codimension 1. Indeed, a weighted projective space is characterized by the property that the divisor class group has no torsion (cf. Lemma 3.1).

Let $N_{X^{\prime}}$ be the sublattice of $N_{X}$ generated by the $v_{i}$. By the equation (2.1), the toric variety $X^{\prime}$ corresponding to the fan $\Delta_{X}$ in $N_{X^{\prime}, \mathbb{R}}$, with the lattice $N_{X^{\prime}}$, is isomorphic to the weighted projective space $\mathbb{P}\left(a_{1}, \ldots, a_{n+1}\right)$. The natural morphism $\bar{\sigma}_{1}: X^{\prime} \rightarrow X$ is etale in codimension 1 . Let $D_{i}^{\prime}$ be the prime divisors on $X^{\prime}$ corresponding to the $v_{i}, \mathcal{X}^{\prime}$ the smooth DeligneMumford stack associated to the pair $\left(X^{\prime}, \sum_{i} \frac{r_{i}-1}{r_{i}} D_{i}^{\prime}\right)$ with the projections $\pi_{X^{\prime}}: \mathcal{X}^{\prime} \rightarrow X^{\prime}$ and $\sigma_{1}: \mathcal{X}^{\prime} \rightarrow \mathcal{X}$, and let $\mathcal{D}_{i}^{\prime}$ be the prime divisors on $\mathcal{X}^{\prime}$ such that $\pi_{X^{\prime}}^{*} D_{i}^{\prime}=r_{i} \mathcal{D}_{i}^{\prime}$.

Let $r$ be a positive integer such that $a_{i} r$ is divisible by $r_{i}$ for any $i$, and let $N_{\tilde{X}}$ be the sublattice of $N_{X}$ generated by the vectors $\tilde{v}_{i}=a_{i} r v_{i}$. We have

$$
\sum_{i=1}^{n+1} \tilde{v}_{i}=0
$$


and the toric variety $\tilde{X}$ corresponding to the fan $\Delta_{X}$ in $N_{\tilde{X}, \mathbb{R}}$, with the lattice $N_{\tilde{X}}$, is isomorphic to the projective space $\mathbb{P}^{n}$. Let $\sigma_{2}: \tilde{X} \rightarrow \mathcal{X}^{\prime}$ be the natural morphism, and set $\sigma=\sigma_{1} \circ \sigma_{2}, \bar{\sigma}_{2}=\pi_{X^{\prime}} \circ \sigma_{2}$ and $\bar{\sigma}=\pi_{X} \circ \sigma$. Let $\tilde{D}_{i}$ be the prime divisors on $\tilde{X}$ corresponding to the vectors $\tilde{v}_{i}$. Moreover, let $N_{X^{\prime \prime}}$ be the sublattice of $N_{X}$ generated by the $r_{i} v_{i}$. We note that the vectors $r_{i} v_{i}$ are not necessarily primitive in this lattice.

Lemma 3.1. (1) A divisor $\sum_{i} k_{i} \mathcal{D}_{i}$ is torsion in the divisor class group of $\mathcal{X}$ if and only if

$$
\sum_{i} \frac{a_{i} k_{i}}{r_{i}}=0
$$

(2) The group of torsion divisor classes on $\mathcal{X}$ is dual to the quotient group $N_{X} / N_{X^{\prime \prime}}$.

(3) The group of torsion Weil divisor classes on $X$ is dual to the quotient group $N_{X} / N_{X^{\prime}}$.

Proof. (1) A divisor $\sum_{i} k_{i} \mathcal{D}_{i}$ is linearly equivalent to 0 if and only if there exists $m \in M_{X}=N_{X}^{*}$ such that $\left(m, r_{i} v_{i}\right)=k_{i}$, because the morphism $\pi_{X}: \mathcal{X} \rightarrow X$ is birational. Thus $\sum_{i} k_{i} \mathcal{D}_{i}$ is torsion if and only if there exists $m \in M_{X, \mathbb{R}}$ such that $\left(m, r_{i} v_{i}\right)=k_{i}$. The latter condition is equivalent to the equality $\sum_{i} \frac{a_{i} k_{i}}{r_{i}}=0$.

(2) For $m \in M_{X, \mathbb{R}}$, we have $\left(m, r_{i} v_{i}\right) \in \mathbb{Z}$ for all $v_{i}$ if and only if $m \in$ $M_{X^{\prime \prime}}=N_{X^{\prime \prime}}^{*}$. Therefore, the group of torsion divisor classes is isomorphic to $M_{X^{\prime \prime}} / M_{X}$.

(3) is a particular case of (2).

Remark 3.2. If $B=0$, i.e., $r_{i}=1$ for all $i$, then the divisor class groups of $X$ and $\mathcal{X}$ are isomorphic.

Example 3.3. Torsion divisor classes correspond to etale coverings of the stack. For example, let $X=\mathbb{P}^{n}$ be the projective space, and $\mathcal{X}$ the smooth stack associated to the pair $\left(X, \frac{r-1}{r} \sum_{i=1}^{n+1} H_{i}\right)$, where the $H_{i}$ are coordinate hyperplanes. Let $\mathcal{H}_{i}$ be the prime divisors on $\mathcal{X}$ above the $H_{i}$ so that $\pi_{X}^{*} H_{i}=$ $r \mathcal{H}_{i}$ for the projection $\pi_{X}: \mathcal{X} \rightarrow X$.

Let $\tilde{X}=\mathbb{P}^{n}$ be another projective space, and let $\sigma: \tilde{X} \rightarrow \mathcal{X}$ be the Kummer covering with Galois group $(\mathbb{Z} / r)^{n}$ obtained by taking the $r$-th roots of the coordinates. Then $\sigma$ is etale, and we have

$$
\sigma_{*} \mathcal{O}_{\tilde{X}} \cong \bigoplus_{l_{1}, \ldots, l_{n}=0}^{r-1} \mathcal{O}_{\mathcal{X}}\left(\sum_{i=1}^{n} l_{i} \mathcal{H}_{i}+\left(-\sum_{i=1}^{n} l_{i}\right) \mathcal{H}_{n+1}\right)
$$


We note that the direct summands are invertible sheaves on $\mathcal{X}$ corresponding to the torsion divisor classes. In the usual language, if we denote $\bar{\sigma}=\pi_{X} \circ \sigma$ : $\tilde{X} \rightarrow X$, then

$$
\bar{\sigma}_{*} \mathcal{O}_{\tilde{X}} \cong \bigoplus_{l_{1}, \ldots, l_{n}=0}^{r-1} \mathcal{O}_{X}\left(-\left\ulcorner\frac{\sum_{i=1}^{n} l_{i}}{r}\right\urcorner H_{n+1}\right)
$$

because

$$
\pi_{X *} \mathcal{O}_{\mathcal{X}}\left(\sum_{i=1}^{n} l_{i} \mathcal{H}_{i}+\left(-\sum_{i=1}^{n} l_{i}\right) \mathcal{H}_{n+1}\right)=\mathcal{O}_{X}\left(-\left\ulcorner\frac{\sum_{i=1}^{n} l_{i}}{r}\right\urcorner H_{n+1}\right) .
$$

More generally, we have

$$
\sigma_{*} \mathcal{O}_{\tilde{X}}(-p) \cong \bigoplus_{l_{1}, \ldots, l_{n}=0}^{r-1} \mathcal{O}_{\mathcal{X}}\left(\sum_{i=1}^{n} l_{i} \mathcal{H}_{i}+\left(-p-\sum_{i=1}^{n} l_{i}\right) \mathcal{H}_{n+1}\right)
$$

and

$$
\bar{\sigma}_{*} \mathcal{O}_{\tilde{X}}(-p) \cong \bigoplus_{l_{1}, \ldots, l_{n}=0}^{r-1} \mathcal{O}_{X}\left(-\left\ulcorner\frac{p+\sum_{i=1}^{n} l_{i}}{r}\right\urcorner H_{n+1}\right) .
$$

For example, the direct images of the sheaves $\mathcal{O}_{\tilde{X}}(-p)$ for $0 \leq p \leq n$, which generates the derived category $D^{b}(\operatorname{Coh}(\tilde{X}))([1])$, have the direct summands of the form $\mathcal{O}_{X}(-q)$ for $0 \leq q \leq n$ (cf. [11]).

Lemma 3.4. (1) Let $G_{1}=N_{X} / N_{X^{\prime}}$ be the Galois group of the covering $\bar{\sigma}_{1}: X^{\prime} \rightarrow X$. Then there is the following decomposition into eigenspaces with respect to the $G_{1}$-action

$$
\sigma_{1 *} \mathcal{O}_{\mathcal{X}^{\prime}}\left(\sum_{i} d_{i} \mathcal{D}_{i}^{\prime}\right) \cong \bigoplus_{k} \mathcal{O}_{\mathcal{X}}\left(\sum_{i}\left(d_{i}+k_{i} r_{i}\right) \mathcal{D}_{i}\right)
$$

where the sequences of integers $k=\left(k_{i}\right)$ in the summation are determined by the equation $k_{i}=\left(m, v_{i}\right)$ for the representatives $m$ of the group of torsion Weil divisor classes $M_{X^{\prime}} / M_{X}$ of $X$.

(2) Let $G_{2}=N_{X^{\prime}} / N_{\tilde{X}}$ be the Galois group of the covering $\bar{\sigma}_{2}: \tilde{X} \rightarrow X^{\prime}$. Then there is the following decomposition into eigenspaces with respect to the $G_{2}$-action

$$
\sigma_{1 *} \mathcal{O}_{\tilde{X}}(-p) \cong \bigoplus_{l} \mathcal{O}_{\mathcal{X}^{\prime}}\left(\sum_{1 \leq i \leq n}\left\llcorner\frac{l_{i} r_{i}}{a_{i} r}\right\lrcorner \mathcal{D}_{i}^{\prime}+\left\llcorner\frac{\left(l_{n+1}-p\right) r_{n+1}}{a_{n+1} r}\right\lrcorner \mathcal{D}_{n+1}^{\prime}-\frac{1}{r} \sum_{i=1}^{n+1} l_{i}\right)
$$


where the sequences of integers $l=\left(l_{i}\right)$ in the summation run under the conditions that $0 \leq l_{i}<a_{i} r$ and $r \mid \sum_{i=1}^{n+1} l_{i}$.

(3) Let $G=N_{X} / N_{\tilde{X}}$ the Galois group of the covering $\bar{\sigma}: \tilde{X} \rightarrow X$. Then there is the following decomposition into eigenspaces with respect to the $G$ action

$$
\sigma_{*} \mathcal{O}_{\tilde{X}}(-p) \cong \bigoplus_{k} \mathcal{O}_{\mathcal{X}}\left(\sum_{1 \leq i \leq n}\left\llcorner\frac{k_{i} r_{i}}{a_{i} r}\right\lrcorner \mathcal{D}_{i}+\left\llcorner\frac{\left(k_{n+1}-p\right) r_{n+1}}{a_{n+1} r}\right\lrcorner \mathcal{D}_{n+1}\right)
$$

where the sequences of integers $k=\left(k_{i}\right)$ satisfy the equation

$$
\sum_{i=1}^{n+1} k_{i}=0
$$

Proof. (1) is clear.

(2) We have an exact sequence

$$
0 \rightarrow \mathbb{Z} / r \rightarrow \bigoplus_{i=1}^{n+1} \mathbb{Z} / a_{i} r \rightarrow G_{2} \rightarrow 0
$$

where 1 in the first term is sent to $\left(a_{i}\right)$ in the second term. Thus

$$
G_{2}^{*} \cong\left\{\left(l_{i}\right) \in \bigoplus_{i=1}^{n+1} \mathbb{Z} / a_{i} r ; \sum_{i=1}^{n+1} l_{i}=0 \quad \bmod r\right\} .
$$

We have $\sigma_{1}^{*} \mathcal{D}_{i}^{\prime}=\frac{a_{i} r}{r_{i}} \tilde{D}_{i}$ for the prime divisor $\tilde{D}_{i}$ on $\tilde{X}$ above $\mathcal{D}_{i}^{\prime}$. Since $\frac{a_{i} l_{i}}{a_{i} r}=\frac{l_{i}}{r}$, we obtain the formula. We note that $\mathcal{O}_{\mathcal{X}^{\prime}}(1)$ is well-defined because $\mathcal{X}^{\prime}$ has no torsion divisor classes.

(3) By combining (1) and (2), we obtain

$$
\begin{aligned}
\sigma_{*} \mathcal{O}_{\tilde{X}}(-p) \cong \bigoplus_{k, l} \mathcal{O}_{\mathcal{X}} & \left(\sum_{1 \leq i \leq n}\left(k_{i} r_{i}+\left\llcorner\frac{l_{i} r_{i}}{a_{i} r}\right\lrcorner\right) \mathcal{D}_{i}\right. \\
& \left.+\left(k_{n+1} r_{n+1}+\left\llcorner\frac{\left(l_{n+1}-p\right) r_{n+1}}{a_{n+1} r}\right\lrcorner\right) \mathcal{D}_{n+1}\right)
\end{aligned}
$$

where the $k=\left(k_{i}\right)$ satisfy the equation

$$
\sum_{i=1}^{n+1} a_{i} k_{i}=-\frac{1}{r} \sum_{i=1}^{n+1} l_{i}
$$

and the summation on $l=\left(l_{i}\right)$ is under the restriction that $0 \leq l_{i}<a_{i} r$ and $r \mid \sum_{i} l_{i}$. If we replace $a_{i} r k_{i}+l_{i}$ by $k_{i}$, then we obtain our assertion. 
Theorem 3.5. (1) An invertible sheaf $\mathcal{O}_{\mathcal{X}}\left(\sum_{i=1}^{n+1} k_{i} \mathcal{D}_{i}\right)$ on $\mathcal{X}$ is an exceptional object for any sequence of integers $k=\left(k_{i}\right)$ for $1 \leq i \leq n+1$.

(2) If $\sum_{i=1}^{n+1} \frac{a_{i} k_{i}}{r_{i}}>\sum_{i=1}^{n+1} \frac{a_{i} k_{i}^{\prime}}{r_{i}}>\sum_{i=1}^{n+1} \frac{a_{i}\left(k_{i}-1\right)}{r_{i}}$, then

$$
\operatorname{Hom}^{q}\left(\mathcal{O}_{\mathcal{X}}\left(\sum_{i=1}^{n+1} k_{i} \mathcal{D}_{i}\right), \mathcal{O}_{\mathcal{X}}\left(\sum_{i=1}^{n+1} k_{i}^{\prime} \mathcal{D}_{i}\right)\right)=0
$$

for all $q$, where $k^{\prime}=\left(k_{i}^{\prime}\right)$ is another sequence of integers.

(3) If $\sum_{i=1}^{n+1} \frac{a_{i} k_{i}}{r_{i}}=\sum_{i=1}^{n+1} \frac{a_{i} k_{i}^{\prime}}{r_{i}}$ and $\sum_{i=1}^{n+1} k_{i} \mathcal{D}_{i} \nsim \sum_{i=1}^{n+1} k_{i}^{\prime} \mathcal{D}_{i}$, then

$$
\operatorname{Hom}^{q}\left(\mathcal{O}_{\mathcal{X}}\left(\sum_{i=1}^{n+1} k_{i} \mathcal{D}_{i}\right), \mathcal{O}_{\mathcal{X}}\left(\sum_{i=1}^{n+1} k_{i}^{\prime} \mathcal{D}_{i}\right)\right)=0
$$

for all $q$.

(4) If $\sum_{i=1}^{n+1} \frac{a_{i} k_{i}}{r_{i}} \leq \sum_{i=1}^{n+1} \frac{a_{i} k_{i}^{\prime}}{r_{i}}$, then

$$
\operatorname{Hom}^{q}\left(\mathcal{O}_{\mathcal{X}}\left(\sum_{i=1}^{n+1} k_{i} \mathcal{D}_{i}\right), \mathcal{O}_{\mathcal{X}}\left(\sum_{i=1}^{n+1} k_{i}^{\prime} \mathcal{D}_{i}\right)\right)=0
$$

for $q \neq 0$.

(5) The set of invertible sheaves $\mathcal{O}_{\mathcal{X}}\left(\sum_{i=1}^{n+1} k_{i} \mathcal{D}_{i}\right)$ for

$$
0 \geq \sum_{i=1}^{n+1} \frac{a_{i} k_{i}}{r_{i}}>-\sum_{i=1}^{n+1} \frac{a_{i}}{r_{i}}
$$

generates the triangulated category $D^{b}(\operatorname{Coh}(\mathcal{X}))$.

Proof. The canonical divisor of $\mathcal{X}$ is given by

$$
\omega_{\mathcal{X}} \cong \pi_{X}^{*} \omega_{X} \otimes \mathcal{O}_{\mathcal{X}}\left(\sum_{i}\left(r_{i}-1\right) \mathcal{D}_{i}\right) \cong \mathcal{O}_{\mathcal{X}}\left(-\sum_{i} \mathcal{D}_{i}\right)
$$

An invertible sheaf $\mathcal{O}_{\mathcal{X}}\left(\sum_{i} k_{i} \mathcal{D}_{i}\right)$ is ample if and only if $\sum_{i} \frac{a_{i} k_{i}}{r_{i}}>0$. Therefore, the assertions (1) through (4) follow immediately from the vanishing theorem $([13])$.

(5) follows from a similar generalization of the Beilinson resolution theorem $([1])$ as in [1] $\S 5$. Indeed, the integral functor corresponding to an object $e$ on $\mathcal{X} \times \mathcal{X}$ given by

$$
\begin{aligned}
e=\{0 & \rightarrow\left[\sigma_{*} \mathcal{O}_{\tilde{X}}(-n) \bigotimes \sigma_{*} \Omega_{\tilde{X}}^{n}(n)\right]^{G} \rightarrow \ldots \\
& \left.\rightarrow\left[\sigma_{*} \mathcal{O}_{\tilde{X}}(-1) \bigotimes \sigma_{*} \Omega_{\tilde{X}}^{1}(1)\right]^{G} \rightarrow\left[\sigma_{*} \mathcal{O}_{\tilde{X}} \otimes \sigma_{*} \mathcal{O}_{\tilde{X}}\right]^{G} \rightarrow 0\right\}
\end{aligned}
$$


is isomorphic to the identity functor, where the group $G$ acts diagonally on the tensor products. Thus the derived category $D^{b}(\mathrm{Coh}(\mathcal{X}))$ is generated by the direct summands of the sheaves $\sigma_{*} \mathcal{O}_{\tilde{X}}(-p)$ for $0 \leq p \leq n$ given in Lemma 3.4 (3).

Since $\sum_{i} k_{i}=0$, we have

$$
\sum_{i=1}^{n} \frac{a_{i} \frac{k_{i} r_{i}}{a_{i} r}}{r_{i}}+\frac{a_{n+1} \frac{\left(k_{n+1}-p\right) r_{n+1}}{a_{n+1} r}}{r_{n+1}}=-\frac{p}{r} .
$$

Then we calculate

$$
\begin{aligned}
& 0 \geq \sum_{i=1}^{n} \frac{a_{i}\left\llcorner\frac{k_{i} r_{i}}{a_{i} r}\right\lrcorner}{r_{i}}+\frac{a_{n+1}\left\llcorner\frac{\left(k_{n+1}-p\right) r_{n+1}}{a_{n+1} r}\right\lrcorner}{r_{n+1}} \\
& \geq-\sum_{i=1}^{n+1} \frac{a_{i}}{r_{i}}\left(1-\frac{1}{s_{i}}\right)-\frac{n}{r} \geq-\sum_{i=1}^{n+1} \frac{a_{i}}{r_{i}}+\frac{1}{r}
\end{aligned}
$$

where we put $a_{i} r=r_{i} s_{i}$ for some integers $s_{i}$.

Corollary 3.6. Let $(X, B)$ be a $\mathbb{Q}$-factorial projective toric variety such that $-\left(K_{X}+B\right)$ is ample, $\rho(X)=1$ and that the coefficients of $B$ belong to the set $\left\{\frac{r-1}{r} ; r \in \mathbb{Z}_{>0}\right\}$. Let $\mathcal{X}$ be the smooth Deligne-Mumford stack associated to the pair $(X, B)$. Then the derived category $D^{b}(C o h(\mathcal{X}))$ has a strong complete exceptional collection consisting of invertible sheaves.

Proof. The number of isomorphism classes of the set of invertible sheaves $\mathcal{O}_{\mathcal{X}}\left(\sum_{i} k_{i} \mathcal{D}_{i}\right)$ for $0 \geq \sum_{i} \frac{a_{i} k_{i}}{r_{i}}>-\sum_{i} \frac{a_{i}}{r_{i}}$ is finite.

\section{Mori fiber space}

We consider a toric Mori fiber space $\phi: X \rightarrow Y$ with respect to $K_{X}+B$. This fibration is not necessarily locally trivial because there may be multiple fibers. But it becomes locally trivial after taking coverings:

Lemma 4.1. Let $Y_{0}$ be an invariant open affine subset of $Y$, and let $X_{0}=$ $\phi^{-1}\left(Y_{0}\right)$. Then there exist finite surjective toric morphisms $\tau_{X_{0}}: X_{0}^{\prime} \rightarrow X_{0}$ and $\tau_{Y_{0}}: Y_{0}^{\prime \prime} \rightarrow Y_{0}$ with a toric surjective morphism $\phi_{0}^{\prime}: X_{0}^{\prime} \rightarrow Y_{0}^{\prime \prime}$ which satisfy the following conditions. 
(1) $\tau_{X_{0}}$ is etale in codimension 1.

(2) $\phi \circ \tau_{X_{0}}=\tau_{Y_{0}} \circ \phi_{0}^{\prime}$.

(3) $X_{0}^{\prime}$ is isomorphic to the direct product of $Y_{0}^{\prime \prime}$ and a weighted projective space, and $\phi_{0}^{\prime}$ corresponds to the projection.

Proof. Let $N_{Y}$ be the lattice of 1-parameter subgroups of the torus for $Y$, and $\Delta_{Y}$ the fan in $N_{Y, \mathbb{R}}$ corresponding to $Y$. We take the wall $w$ described in the formula (2.1) such that the corresponding extremal rational curve is contained in $X_{0}$. We have

$$
N_{Y}=N_{X} /\left(\bigoplus_{i=1}^{\alpha} \mathbb{R} v_{i} \cap N_{X}\right) .
$$

Let $h: N_{X} \rightarrow N_{Y}$ be the projection. We write $h\left(v_{i}\right)=s_{i} \bar{v}_{i}$ for primitive vectors $\bar{v}_{i}$ in $N_{Y}$ and positive integers $s_{i}$ for $\alpha+1 \leq i \leq n+1$. Then these $\bar{v}_{i}$ give the set of edges of an $(n+1-\alpha)$-dimensional cone $\sigma_{0}$ in $\Delta_{Y}$ corresponding to $Y_{0}$. Let $E_{i}$ be the prime divisors on $Y$ corresponding to the vectors $\bar{v}_{i} . X_{0}$ coincides with the toric variety corresponding to the fan $\Delta_{X} \cap h^{-1}\left(\sigma_{0}\right)$ in $N_{X, \mathbb{R}}$.

Let $N_{X_{0}^{\prime}}$ be the sublattice of $N_{X}$ generated by the $v_{i}$ for $1 \leq i \leq n+1$, and $N_{Y_{0}^{\prime}}\left(\right.$ resp. $\left.N_{Y_{0}^{\prime \prime}}\right)$ of $N_{Y}$ generated by the $\bar{v}_{i}\left(\right.$ resp. $\left.h\left(v_{i}\right)\right)$ for $\alpha+1 \leq i \leq n+1$. Let $X_{0}^{\prime}$ be the toric variety corresponding to the fan $\Delta_{X} \cap h^{-1}\left(\sigma_{0}\right)$ in $N_{X_{0}^{\prime}, \mathbb{R}}$, and $Y_{0}^{\prime}$ (resp. $Y_{0}^{\prime \prime}$ ) the one corresponding to the cone $\sigma_{0}$ in $N_{Y_{0}^{\prime}, \mathbb{R}}$ (resp.

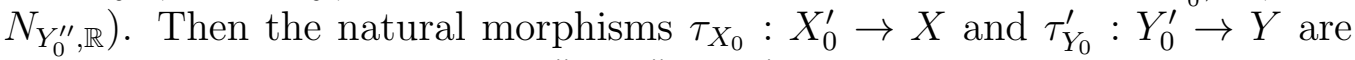
etale in codimension 1, while $\tau_{Y_{0}}^{\prime \prime}: Y_{0}^{\prime \prime} \rightarrow Y_{0}^{\prime}$ is not in general. Since

$$
\sum_{i=1}^{\alpha} a_{i} v_{i}=0
$$

$X_{0}^{\prime}$ is isomorphic to the product of $Y_{0}^{\prime \prime}$ with a weighted projective space $\mathbb{P}\left(a_{1}, \ldots, a_{\alpha}\right)$.

We define the boundary $\mathbb{Q}$-divisor $C$ on $Y$ by assigning coefficients $\frac{r_{i} s_{i}-1}{r_{i} s_{i}}$ to the irreducible components $E_{i}$, where the $s_{i}$ are defined in the proof of the above lemma. We note that, even if we start with the non-boundary case $B=0$, the naturally defined boundary divisor $C$ on $Y$ is non-zero in general, because there may be multiple fibers for $\phi$. Let $\mathcal{Y}$ be the smooth DeligneMumford stack associated to the pair $(Y, C)$. The above lemma implies the following: 
Corollary 4.2. The natural morphism $\psi: \mathcal{X} \rightarrow \mathcal{Y}$ is smooth.

Theorem 4.3. (1) The functor $\psi^{*}: D^{b}(\operatorname{Coh}(\mathcal{Y})) \rightarrow D^{b}(\operatorname{Coh}(\mathcal{X}))$ is fully faithful.

Let $D^{b}(\operatorname{Coh}(\mathcal{Y}))_{k}$ denote the full subcategory of $D^{b}(\operatorname{Coh}(\mathcal{X}))$ defined by

$$
D^{b}(\operatorname{Coh}(\mathcal{Y}))_{k}=\psi^{*} D^{b}(\operatorname{Coh}(\mathcal{Y})) \otimes \mathcal{O}_{\mathcal{X}}\left(\sum_{i=1}^{\alpha} k_{i} \mathcal{D}_{i}\right)
$$

for a sequence of integers $k=\left(k_{i}\right)$ for $1 \leq i \leq \alpha$.

(2) If $\sum_{i=1}^{\alpha} \frac{a_{i} k_{i}}{r_{i}}>\sum_{i=1}^{\alpha} \frac{a_{i} k_{i}^{\prime}}{r_{i}}>\sum_{i=1}^{\alpha} \frac{a_{i}\left(k_{i}-1\right)}{r_{i}}$, then

$$
\operatorname{Hom}^{q}\left(D^{b}(\operatorname{Coh}(\mathcal{Y}))_{k}, D^{b}(\operatorname{Coh}(\mathcal{Y}))_{k^{\prime}}\right)=0
$$

for all $q$, where $k^{\prime}=\left(k_{i}^{\prime}\right)$ is another sequence of integers. then

(3) If $\sum_{i=1}^{\alpha} \frac{a_{i} k_{i}}{r_{i}}=\sum_{i=1}^{\alpha} \frac{a_{i} k_{i}^{\prime}}{r_{i}}$ and $\mathcal{O}_{\mathcal{X}}\left(\sum_{i=1}^{\alpha}\left(k_{i}-k_{i}^{\prime}\right) \mathcal{D}_{i}\right) \notin \psi^{*} D^{b}(\operatorname{Coh}(\mathcal{Y}))$,

$$
\operatorname{Hom}^{q}\left(D^{b}(\operatorname{Coh}(\mathcal{Y}))_{k}, D^{b}(\operatorname{Coh}(\mathcal{Y}))_{k^{\prime}}\right)=0
$$

for all $q$.

(4) The set of subcategories $D^{b}(\operatorname{Coh}(\mathcal{Y}))_{k}$ for

$$
0 \geq \sum_{i=1}^{\alpha} \frac{a_{i} k_{i}}{r_{i}}>-\sum_{i=1}^{\alpha} \frac{a_{i}}{r_{i}}
$$

generates the triangulated category $D^{b}(\operatorname{Coh}(\mathcal{X}))$.

Proof. (1) By [4] or [5], it is sufficient to prove the following statement; if $A$ and $B$ are skyscraper sheaves on $\mathcal{Y}$ of length 1 , then the natural homomorphism $\operatorname{Hom}^{p}(A, B) \rightarrow \operatorname{Hom}^{p}\left(\psi^{*} A, \psi^{*} B\right)$ is bijective. This follows from the fact that $X_{0}^{\prime}$ is isomorphic to the product of $Y_{0}^{\prime \prime}$ with a weighted projective space $\mathbb{P}\left(a_{1}, \ldots, a_{\alpha}\right)$, and that the natural homomorphism of Galois groups $N_{X} / N_{X_{0}^{\prime}} \rightarrow N_{Y} / N_{Y_{0}^{\prime \prime}}$ is surjective .

For (2) and (3), we use a spectral sequence

$$
\begin{aligned}
& E_{2}^{p, q}=H^{p}\left(\mathcal{Y}, \mathcal{H o m}(A, B) \otimes R^{q} \psi_{*} \mathcal{O}_{\mathcal{X}}\left(\sum_{i=1}^{\alpha} k_{i} \mathcal{D}_{i}\right)\right) \\
& \Rightarrow \operatorname{Hom}^{p+q}\left(\psi^{*} A, \psi^{*} B \otimes \mathcal{O}_{\mathcal{X}}\left(\sum_{i=1}^{\alpha} k_{i} \mathcal{D}_{i}\right)\right)
\end{aligned}
$$


for invertible sheaves $A, B$ on $\mathcal{Y}$. The direct image sheaves vanish in our case, because the relative canonical divisor for $\psi$ is given by

$$
\omega_{\mathcal{X} / \mathcal{Y}} \cong \mathcal{O}_{\mathcal{X}}\left(-\sum_{i=1}^{\alpha} \mathcal{D}_{i}\right)
$$

and an invertible sheaf $\mathcal{O}_{\mathcal{X}}\left(\sum_{i=1}^{\alpha} k_{i} \mathcal{D}_{i}\right)$ is $\psi$-ample if and only if $\sum_{i=1}^{\alpha} \frac{a_{i} k_{i}}{r_{i}}>0$ ([13]).

(4) In general, a full triangulated subcategory $\mathcal{B}$ of a triangulated category $\mathcal{A}$ is said to be right (resp. left) admissible if $\mathcal{A}$ is generated by $\mathcal{B}$ and $\mathcal{B}^{\perp}$ (resp. $\mathcal{B}$ and ${ }^{\perp} \mathcal{B}$ ), where $\mathcal{B}^{\perp}$ (resp. ${ }^{\perp} \mathcal{B}$ ) denotes the right (resp. left) orthogonal complement of $\mathcal{B}$ in $\mathcal{A}([3])$. The subcategory triangulated $\mathcal{T}$ of $D^{b}(\operatorname{Coh}(\mathcal{X}))$ generated by the subcategories $D^{b}(\operatorname{Coh}(\mathcal{Y}))_{k}$ is admissible by loc. cit. 1.12, 2.6 and 2.11. Therefore, it is sufficient to prove that the left orthogonal $\perp \mathcal{T}$ consists of 0 objects.

Let $A$ be an arbitrary skyscraper sheaf of length 1 on $\mathcal{X}$ supported at a point $P$. Then by Theorem [3.6, there exists a skyscraper sheaf $B$ of length 1 on $\mathcal{Y}$ supported at $Q=\phi(P)$ such that $A$ is contained in the subcategory generated by the sheaves of the form $\psi^{*} B \otimes \mathcal{O}_{\mathcal{X}}\left(\sum_{i=1}^{\alpha} k_{i} \mathcal{D}_{i}\right)$ for $0 \geq \sum_{i=1}^{\alpha} \frac{a_{i} k_{i}}{r_{i}}>-\sum_{i=1}^{\alpha} \frac{a_{i}}{r_{i}}$. Thus $A$ contained in $\mathcal{T}$. Hence ${ }^{\perp} \mathcal{T}=0$, because such $A \operatorname{span} D^{b}(\operatorname{Coh}(\mathcal{X}))$ ([4] or [5]).

Corollary 4.4. Assume that $D^{b}($ Coh $(\mathcal{Y}))$ has a complete exceptional collection consisting of sheaves. Then so has $D^{b}(\operatorname{Coh}(\mathcal{X}))$.

\section{Divisorial contraction}

We consider a toric divisorial contraction $\phi: X \rightarrow Y . K_{X}+B$ is negative for $\phi$, and $C=\phi_{*} B$ is the strict transform. Let $D$ be the exceptional divisor of the contraction. Then the restriction $\bar{\phi}: D \rightarrow F=\phi(D)$ is a Mori fiber space which was treated in the previous section.

Let $\mathcal{Y}$ be the stack associated to the pair $(Y, C)$. We note that there is no morphism of stacks from $\mathcal{X}$ to $\mathcal{Y}$ in general. But there is still a fully faithful functor $\Phi: D^{b}(\operatorname{Coh}(\mathcal{Y})) \rightarrow D^{b}(\operatorname{Coh}(\mathcal{X}))$ by 12 Theorem $4.2(2)$. Indeed, let $\mathcal{W}$ be the normalization of the fiber product $\mathcal{X} \times_{Y} \mathcal{Y}$, and let $\mu: \mathcal{W} \rightarrow \mathcal{X}$ and $\nu: \mathcal{W} \rightarrow \mathcal{Y}$ be the projections. Then $\Phi=\mu_{*} \circ \nu^{*}$ is fully faithful. We regard $D^{b}(\operatorname{Coh}(\mathcal{Y}))$ as a full subcategory of $D^{b}(\operatorname{Coh}(\mathcal{X}))$ through this functor. 
Let $E_{i}=\phi_{*} D_{i}$ be the prime divisors on $Y$ corresponding to the edges $v_{i}$ for $1 \leq i \leq n$. Those $E_{i}$ for $1 \leq i \leq \alpha$ are the divisors which contain the center $F$ of the blowing-up $\phi$, and $D=D_{n+1}$ is the exceptional divisor. Let $\mathcal{E}_{i}$ be the prime divisors on $\mathcal{Y}$ corresponding to the $E_{i}$. The following formula is proved in the proof of [12] Theorem 4.2 (2):

$$
\begin{aligned}
& \Phi\left(\mathcal{O}_{\mathcal{Y}}\left(\sum_{i=1}^{n} k_{i} \mathcal{E}_{i}\right)\right) \cong \mathcal{O}_{\mathcal{X}}\left(\sum_{i=1}^{n+1} k_{i} \mathcal{D}_{i}\right) \\
& k_{n+1}=\left\llcorner\frac{r_{n+1}}{b_{n+1}} \sum_{i=1}^{n} \frac{a_{i} k_{i}}{r_{i}}\right\lrcorner
\end{aligned}
$$

for any integers $k_{i}$ for $1 \leq i \leq n$, where we put $b_{n+1}=-a_{n+1}>0$.

Let $r$ be a positive integer such that $a_{i} r$ is divisible by $r_{i}$ for $1 \leq i \leq n+1$. We set

$$
\left|a_{i}\right| \cdot r=r_{i} s_{i} .
$$

Let $s=\left(s_{1}, \ldots, s_{n+1}\right)$ be the greatest common divisor, and set $s_{i}=s \bar{s}_{i}$. Then the fractional part of the rational number

$$
\frac{r_{n+1}}{b_{n+1}} \sum_{i=1}^{n} \frac{a_{i} k_{i}}{r_{i}}=\frac{\sum_{i=1}^{n} k_{i} \bar{s}_{i}}{\bar{s}_{n+1}}
$$

can take arbitrary value in the set $\left\{0, \frac{1}{\bar{s}_{n+1}}, \ldots, \frac{\bar{s}_{n+1}-1}{\bar{s}_{n+1}}\right\}$ when we vary the sequence $k$, because $\left(\bar{s}_{1}, \ldots, \bar{s}_{n+1}\right)=1$.

The Mori fiber space $\bar{\phi}: D \rightarrow F$ is described as follows. The lattice of 1-parameter subgroup for $D$ is given by $\bar{N}=N_{X} / \mathbb{Z} v_{n+1}$. We write $v_{i} \bmod \mathbb{Z} v_{n+1}=t_{i} \bar{v}_{i}$ for $1 \leq i \leq n$, where the $t_{i}$ are positive integers and the $\bar{v}_{i}$ are primitive vectors in $\overline{\bar{N}}$. Let $t=\left(a_{1} t_{1}, \ldots, a_{n} t_{n}\right)$ be the greatest common divisor, and denote $a_{i} t_{i}=t \bar{a}_{i}$. Then we have an equation

$$
\bar{a}_{1} \bar{v}_{1}+\cdots+\bar{a}_{n} \bar{v}_{n}=0 \text {. }
$$

We define a $\mathbb{Q}$-divisor $\bar{B}$ on $D$ by putting coefficients $\frac{r_{i} t_{i}-1}{r_{i} t_{i}}$ to the prime divisors $\bar{D}_{i}=D_{i} \cap D$ for $1 \leq i \leq n$. We also define a $\mathbb{Q}$-divisor $\bar{C}$ on the base space of the Mori fiber space $F$ using $\bar{B}$ as in the previous section. Let $\mathcal{D}$ and $\mathcal{F}$ be the smooth stacks associated to the pairs $(D, \bar{B})$ and $(F, \bar{C})$, respectively. Then there are induced morphisms of stacks $\bar{\psi}: \mathcal{D} \rightarrow \mathcal{F}$ and 
$j: \mathcal{D} \rightarrow \mathcal{X}$. Let $\overline{\mathcal{D}}_{i}$ be the prime divisors on $\mathcal{D}$ corresponding to the $\bar{D}_{i}$ for $1 \leq i \leq n$. Then we have

$$
j^{*} \mathcal{O}_{\mathcal{X}}\left(\mathcal{D}_{i}\right) \cong \mathcal{O}_{\mathcal{D}}\left(\overline{\mathcal{D}}_{i}\right) .
$$

We note that $\left.D_{i}\right|_{D}=\frac{1}{t_{i}} \bar{D}_{i}$ in the usual language.

Remark 5.1. If $r_{n+1}>1$, then the action of the stabilizer group at the generic point of $\mathcal{D}_{n+1}$ is non-trivial. Hence we have $j^{*} \mathcal{O}_{\mathcal{X}}\left(k \mathcal{D}_{n+1}\right)=0$ on $\mathcal{D}$ if $k$ is not divisible by $r_{n+1}$. Indeed, we have

$$
\operatorname{Hom}\left(j^{*} \mathcal{O}_{\mathcal{X}}\left(k \mathcal{D}_{n+1}\right), A\right) \cong \operatorname{Hom}\left(\mathcal{O}_{\mathcal{X}}\left(k \mathcal{D}_{n+1}\right), j_{*} A\right) \cong 0
$$

for any sheaf $A$ on $\mathcal{D}$ in this case.

For example, let $X$ be an affine line with a point $P$, and $\mathcal{X}$ the stack associated to the pair $\left(X, \frac{r-1}{r} P\right)$ with a point $\mathcal{P}$ above $P$. Then we have $j^{*} \mathcal{O}_{\mathcal{X}}(k \mathcal{P})=0$ if $k$ is not divisible by $r$, where $j: P \rightarrow \mathcal{X}$ is the natural morphism. From a resolution

$$
0 \rightarrow \mathcal{O}_{\mathcal{X}}((k-1) \mathcal{P}) \rightarrow \mathcal{O}_{\mathcal{X}}(k \mathcal{P}) \rightarrow \mathcal{O}_{\mathcal{P}}(k \mathcal{P}) \rightarrow 0
$$

it follows that $L_{q} j^{*} \mathcal{O}_{\mathcal{P}}(k \mathcal{P})$ is isomorphic to $\mathcal{O}_{P}$ if $q=0$ and $k \equiv 0 \bmod r$, or $q=1$ and $k \equiv 1 \bmod r$, and 0 if otherwise. Thus

$$
\begin{aligned}
& \operatorname{Hom}^{q}\left(\mathcal{O}_{\mathcal{P}}(k \mathcal{P}), \mathcal{O}_{\mathcal{P}}\right) \cong \operatorname{Hom}^{q}\left(\mathcal{O}_{\mathcal{P}}(k \mathcal{P}), j_{*} \mathcal{O}_{P}\right) \\
& \cong \operatorname{Hom}^{q}\left(L j^{*} \mathcal{O}_{\mathcal{P}}(k \mathcal{P}), \mathcal{O}_{P}\right)
\end{aligned}
$$

is non zero if and only if $q=0$ and $k \equiv 0 \bmod r$, or $q=1$ and $k \equiv 1 \bmod r$.

Theorem 5.2. (1) The functor $j_{*} \bar{\psi}^{*}: D^{b}(\operatorname{Coh}(\mathcal{F})) \rightarrow D^{b}(\operatorname{Coh}(\mathcal{X}))$ is fully faithful.

Let $D^{b}(\operatorname{Coh}(\mathcal{F}))_{k}$ denote the full subcategory of $D^{b}(\operatorname{Coh}(\mathcal{X}))$ defined by

$$
D^{b}(\operatorname{Coh}(\mathcal{F}))_{k}=j_{*} \bar{\psi}^{*} D^{b}(\operatorname{Coh}(\mathcal{F})) \otimes \mathcal{O}_{\mathcal{X}}\left(\sum_{i=1}^{n+1} k_{i} \mathcal{D}_{i}\right)
$$

for a sequence of integers $k=\left(k_{i}\right)$ for $1 \leq i \leq n+1$.

(2) If $0>\sum_{i=1}^{n+1} \frac{a_{i} k_{i}}{r_{i}} \geq-\sum_{i=1}^{n+1} \frac{a_{i}}{r_{i}}$, then

$$
\operatorname{Hom}^{q}\left(\Phi\left(D^{b}(\operatorname{Coh}(\mathcal{Y}))\right), D^{b}(\operatorname{Coh}(\mathcal{F}))_{k}\right)=0
$$


for all $q$.

(3) If $\sum_{i=1}^{n+1} \frac{a_{i} k_{i}}{r_{i}}>\sum_{i=1}^{n+1} \frac{a_{i} k_{i}^{\prime}}{r_{i}}>\sum_{i=1}^{n+1} \frac{a_{i}\left(k_{i}-1\right)}{r_{i}}$, then

$$
\operatorname{Hom}^{q}\left(D^{b}(\operatorname{Coh}(\mathcal{F}))_{k}, D^{b}(\operatorname{Coh}(\mathcal{F}))_{k^{\prime}}\right)=0
$$

for all $q$, where $k^{\prime}=\left(k_{i}^{\prime}\right)$ is another sequence of integers.

(4) If $\sum_{i=1}^{n+1} \frac{a_{i} k_{i}}{r_{i}}=\sum_{i=1}^{n+1} \frac{a_{i} k_{i}^{\prime}}{r_{i}}$, but if $j^{*} \mathcal{O}_{\mathcal{X}}\left(\sum_{i=1}^{n+1}\left(k_{i}-k_{i}^{\prime}\right) \mathcal{D}_{i}\right)=0$ or

$$
j^{*} \mathcal{O}_{\mathcal{X}}\left(\sum_{i=1}^{n+1}\left(k_{i}-k_{i}^{\prime}\right) \mathcal{D}_{i}\right) \notin \bar{\psi}^{*} D^{b}(\operatorname{Coh}(\mathcal{F}))
$$

then

$$
\operatorname{Hom}^{q}\left(D^{b}(\operatorname{Coh}(\mathcal{F}))_{k}, D^{b}(\operatorname{Coh}(\mathcal{F}))_{k^{\prime}}\right)=0
$$

for all $q$.

(5) The subcategories $\Phi\left(D^{b}(\operatorname{Coh}(\mathcal{Y}))\right)$ and the $D^{b}(\operatorname{Coh}(\mathcal{F}))_{k}$ for

$$
0>\sum_{i=1}^{n+1} \frac{a_{i} k_{i}}{r_{i}} \geq-\sum_{i=1}^{n+1} \frac{a_{i}}{r_{i}}
$$

generate the triangulated category $D^{b}(\operatorname{Coh}(\mathcal{X}))$.

Proof. (1) It is sufficient to prove that the natural homomorphism

$$
\operatorname{Hom}^{q}\left(L, L^{\prime}\right) \rightarrow \operatorname{Hom}^{q}\left(j_{*} \bar{\psi}^{*} L, j_{*} \bar{\psi}^{*} L^{\prime}\right)
$$

is bijective for all $q$ and all locally free sheaves $L$ and $L^{\prime}$ on $\mathcal{F}$, because these sheaves span the category $D^{b}(\operatorname{Coh}(\mathcal{F}))$.

We have an exact sequence

$$
0 \rightarrow \mathcal{O}_{\mathcal{X}}\left(-\mathcal{D}_{n+1}\right) \rightarrow \mathcal{O}_{\mathcal{X}} \rightarrow \mathcal{O}_{\mathcal{D}_{n+1}} \rightarrow 0
$$

with an isomorphism $\mathcal{O}_{\mathcal{D}_{n+1}} \cong j_{*} \mathcal{O}_{\mathcal{D}}$. Hence

$$
L_{q} j^{*} j_{*} \mathcal{O}_{\mathcal{D}} \cong \begin{cases}\mathcal{O}_{\mathcal{D}} & \text { for } q=0 \\ j^{*} \mathcal{O}_{\mathcal{X}}\left(-\mathcal{D}_{n+1}\right) & \text { for } q=1 \\ 0 & \text { otherwise }\end{cases}
$$

where $j^{*} \mathcal{O}_{\mathcal{X}}\left(-\mathcal{D}_{n+1}\right)$ is an invertible sheaf on $\mathcal{D}$ if $r_{n+1}=1$, and zero otherwise. 
If $r_{n+1}>1$, then

$$
\begin{aligned}
& \operatorname{Hom}^{q}\left(j_{*} \bar{\psi}^{*} L, j_{*} \bar{\psi}^{*} L^{\prime}\right) \cong \operatorname{Hom}^{q}\left(L j^{*} j_{*} \bar{\psi}^{*} L, \bar{\psi}^{*} L^{\prime}\right) \\
& \cong \operatorname{Hom}^{q}\left(\bar{\psi}^{*} L, \bar{\psi}^{*} L^{\prime}\right) \cong \operatorname{Hom}^{q}\left(L, L^{\prime}\right)
\end{aligned}
$$

as required. If $r_{n+1}=1$, then we know that $j^{*} \mathcal{O}_{\mathcal{X}}\left(\mathcal{D}_{n+1}\right)$ is negative for $\bar{\psi}$, while $j^{*} \mathcal{O}_{\mathcal{X}}\left(\sum_{i=1}^{n+1} \mathcal{D}_{n+1}\right)$ is ample for $\bar{\psi}$, because $\sum_{i=1}^{n+1} \frac{a_{i}}{r_{i}}>0$. Since

$$
\omega_{\mathcal{D} / \mathcal{F}} \cong \mathcal{O}_{\mathcal{D}}\left(-\sum_{i=1}^{\alpha} \overline{\mathcal{D}}_{i}\right) \cong j^{*} \mathcal{O}_{\mathcal{X}}\left(-\sum_{i=1}^{\alpha} \mathcal{D}_{i}\right)
$$

we calculate

$$
\operatorname{Hom}^{q}\left(L_{1} j^{*} j_{*} \bar{\psi}^{*} L, \bar{\psi}^{*} L^{\prime}\right) \cong \operatorname{Hom}^{q}\left(\bar{\psi}^{*} L, \bar{\psi}^{*} L^{\prime} \otimes j^{*} \mathcal{O}_{\mathcal{X}}\left(\mathcal{D}_{n+1}\right)\right) \cong 0
$$

by the relative vanishing theorem for $\bar{\psi}([13])$. Therefore, we have also our assertion in this case.

(2) It is sufficient to prove

$$
\operatorname{Hom}^{q}\left(\mathcal{O}_{\mathcal{X}}\left(\sum_{i=1}^{n+1} k_{i}^{\prime} \mathcal{D}_{i}\right), j_{*} \bar{\psi}^{*} A \otimes \mathcal{O}_{\mathcal{X}}\left(\sum_{i=1}^{n+1} k_{i} \mathcal{D}_{i}\right)\right)=0
$$

for all integers $q$, all sheaves $A$ on $\mathcal{F}$, and for the sequences $(k)$ and $\left(k^{\prime}\right)$ under the additional conditions that

$$
\begin{aligned}
& k_{n+1}^{\prime}=\left\llcorner\frac{r_{n+1}}{b_{n+1}} \sum_{i=1}^{n} \frac{a_{i} k_{i}^{\prime}}{r_{i}}\right\lrcorner \\
& 0>\sum_{i=1}^{n+1} \frac{a_{i} k_{i}}{r_{i}} \geq-\sum_{i=1}^{n+1} \frac{a_{i}}{r_{i}} .
\end{aligned}
$$

By the first condition, we have

$$
0 \leq \sum_{i=1}^{n+1} \frac{a_{i} k_{i}^{\prime}}{r_{i}}<\frac{b_{n+1}}{r_{n+1}}
$$

Hence

$$
0>\sum_{i=1}^{n+1} \frac{a_{i}\left(k_{i}-k_{i}^{\prime}\right)}{r_{i}}>-\sum_{i=1}^{n} \frac{a_{i}}{r_{i}}
$$


By the relative vanishing theorem for $\bar{\psi}$, we have

$$
\begin{aligned}
& \operatorname{Hom}^{q}\left(\mathcal{O}_{\mathcal{X}}\left(\sum_{i=1}^{n+1} k_{i}^{\prime} \mathcal{D}_{i}\right), j_{*} \bar{\psi}^{*} A \otimes \mathcal{O}_{\mathcal{X}}\left(\sum_{i=1}^{n+1} k_{i} \mathcal{D}_{i}\right)\right) \\
& \cong \operatorname{Hom}^{q}\left(j^{*} \mathcal{O}_{\mathcal{X}}\left(\sum_{i=1}^{n+1}\left(k_{i}^{\prime}-k_{i}\right) \mathcal{D}_{i}\right), \bar{\psi}^{*} A\right) \cong 0
\end{aligned}
$$

(3) is similarly proved as in (1). Since $0>\sum_{i=1}^{n+1} \frac{a_{i}\left(k_{i}^{\prime}-k_{i}\right)}{r_{i}}>-\sum_{i=1}^{n} \frac{a_{i}}{r_{i}}+$ $\frac{b_{n+1}}{r_{n+1}}$, we have

$$
R \bar{\psi}_{*} j^{*} \mathcal{O}_{\mathcal{X}}\left(\sum_{i=1}^{n+1}\left(k_{i}^{\prime}-k_{i}\right) \mathcal{D}_{i}\right)=R \bar{\psi}_{*} j^{*} \mathcal{O}_{\mathcal{X}}\left(\mathcal{D}_{n+1}+\sum_{i=1}^{n+1}\left(k_{i}^{\prime}-k_{i}\right) \mathcal{D}_{i}\right)=0
$$

by the relative vanishing theorem for $\bar{\psi}$. Thus

$$
\operatorname{Hom}^{q}\left(j_{*} \bar{\psi}^{*} L, j_{*} \bar{\psi}^{*} L^{\prime} \otimes \mathcal{O}_{\mathcal{X}}\left(\sum_{i=1}^{n+1}\left(k_{i}^{\prime}-k_{i}\right) \mathcal{D}_{i}\right)\right)=0
$$

for all $q$ and all locally free sheaves $L$ and $L^{\prime}$ on $\mathcal{F}$.

(4) is similar to (3).

(5) We shall prove that the left orthogonal $\perp \mathcal{T}$ to the triangulated subcategory $\mathcal{T}$ of $D^{b}(\mathrm{Coh}(\mathcal{X}))$ generated by these subcategories consists of 0 objects as in the proof of Theorem 4.3.

Let $A$ be an arbitrary skyscraper sheaf of length 1 on $\mathcal{X}$ supported at a point $P$. If $P \notin \mathcal{D}_{n+1}$, then $A \in \mathcal{T}$. Otherwise, there is a point $\bar{P}$ on $\mathcal{D}$ such that $P=j(\bar{P})$. Then by Theorem 3.6, there exists a skyscraper sheaf $B$ of length 1 on $\mathcal{F}$ supported at $\bar{Q}=\bar{\psi}(\bar{P})$ such that $A$ is contained in the subcategory generated by the sheaves of the form $j_{*} \bar{\psi}^{*} B \otimes \mathcal{O}_{\mathcal{X}}\left(\sum_{i=1}^{n+1} k_{i} \mathcal{D}_{i}\right)$ for

$$
\frac{b_{n+1}}{r_{n+1}}>\sum_{i=1}^{n+1} \frac{a_{i} k_{i}}{r_{i}} \geq-\sum_{i=1}^{n+1} \frac{a_{i}}{r_{i}}
$$

If $\frac{b_{n+1}}{r_{n+1}}>\sum_{i=1}^{n+1} \frac{a_{i} k_{i}}{r_{i}} \geq 0$, then it follows that $k_{n+1}=\left\llcorner\frac{r_{n+1}}{b_{n+1}} \sum_{i=1}^{n} \frac{a_{i} k_{i}}{r_{i}}\right\lrcorner$. Therefore, $A$ contained in $\mathcal{T}$, hence ${ }^{\perp} \mathcal{T}=0$.

Corollary 5.3. Assume that $D^{b}($ Coh $(\mathcal{Y}))$ has a complete exceptional collection consisting of sheaves. Then so has $D^{b}(\operatorname{Coh}(\mathcal{X}))$. 


\section{$6 \quad$ Log flip}

We consider a toric small contraction $\phi: X \rightarrow Y$ with the $\log$ flip $\phi^{+}$: $X^{+} \rightarrow Y$. $K_{X}+B$ is negative for $\phi$, and $K_{X^{+}}+B^{+}$is ample for $\phi^{+}$, where $B^{+}=\left(\phi_{*}^{+}\right)^{-1} \phi_{*} B$ is the strict transform. The argument for log flips in this section is surprizingly similar to that for the divisorial contractions in the previous section.

Let $\mathcal{X}^{+}$be the smooth Deligne-Mumford stack associated to the pair $\left(X^{+}, B^{+}\right)$. Then there is a fully faithful functor $\Phi: D^{b}\left(\operatorname{Coh}\left(\mathcal{X}^{+}\right)\right) \rightarrow$ $D^{b}(\operatorname{Coh}(\mathcal{X}))$ by [12] Theorem $4.2(3)$. Indeed, let $\mathcal{W}$ be the normalization of the fiber product $\mathcal{X} \times_{Y} \mathcal{X}^{+}$, and let $\mu: \mathcal{W} \rightarrow \mathcal{X}$ and $\nu: \mathcal{W} \rightarrow \mathcal{X}^{+}$be the projections. Then $\Phi=\mu_{*} \circ \nu^{*}$ is fully faithful. We regard $D^{b}\left(\operatorname{Coh}\left(\mathcal{X}^{+}\right)\right)$as a full subcategory of $D^{b}(\operatorname{Coh}(\mathcal{X}))$ through this functor.

Let $D_{i}^{+}=\left(\phi_{*}^{+}\right)^{-1} \phi_{*} D_{i}$ be the prime divisors on $X^{+}$corresponding to the edges $v_{i}$ for $1 \leq i \leq n+1$, and let $\mathcal{D}_{i}^{+}$be the corresponding prime divisors on $\mathcal{X}^{+}$. The following formula is proved in the proof of [12] Theorem 4.2 (3):

$$
\Phi\left(\mathcal{O}_{\mathcal{X}^{+}}\left(\sum_{i=1}^{n+1} k_{i} \mathcal{D}_{i}^{+}\right)\right) \cong \mathcal{O}_{\mathcal{X}}\left(\sum_{i=1}^{n+1} k_{i} \mathcal{D}_{i}\right)
$$

if

$$
0 \leq \sum_{i=1}^{n+1} \frac{a_{i} k_{i}}{r_{i}}<\sum_{i=\beta+1}^{n+1} \frac{b_{i}}{r_{i}}
$$

where we put $b_{i}=-a_{i}$ for $\beta+1 \leq i \leq n+1$.

Let $D$ be the exceptional locus of the contraction $\phi$. Then we have $D=$ $\cap_{i=\beta+1}^{n+1} D_{i}$, and the restriction $\bar{\phi}: D \rightarrow F=\phi(D)$ is a Mori fiber space, which is described as follows. The lattice of 1-parameter subgroup for $D$ is given by $\bar{N}=N_{X} / \bigoplus_{i=\beta+1}^{n+1} \mathbb{Z} v_{i}$. We write $v_{i} \bmod \bigoplus_{i=\beta+1}^{n+1} \mathbb{Z} v_{i}=t_{i} \bar{v}_{i}$ for $1 \leq i \leq \beta$, where the $t_{i}$ are positive integers and the $\bar{v}_{i}$ are primitive vectors in $\bar{N}$. Let $t=\left(a_{1} t_{1}, \ldots, a_{\beta} t_{\beta}\right)$ be the greatest common divisor and $a_{i} t_{i}=t \bar{a}_{i}$. Then we have an equation

$$
\bar{a}_{1} \bar{v}_{1}+\cdots+\bar{a}_{\beta} \bar{v}_{\beta}=0 .
$$

We define a $\mathbb{Q}$-divisor $\bar{B}$ on $D$ by putting coefficients $\frac{r_{i} t_{i}-1}{r_{i} t_{i}}$ to the prime divisors $\bar{D}_{i}=D_{i} \cap D$ for $1 \leq i \leq \beta$. We also define a $\mathbb{Q}$-divisor $\bar{C}$ on the base space of the Mori fiber space $F$ using $\bar{B}$ as before. Let $\mathcal{D}$ and $\mathcal{F}$ be the smooth Deligne-Mumford stacks associated to the pairs $(D, \bar{B})$ and $(F, \bar{C})$, respectively. Then there are induced morphisms of stacks $\bar{\psi}: \mathcal{D} \rightarrow \mathcal{F}$ and 
$j: \mathcal{D} \rightarrow \mathcal{X}$. Let $\overline{\mathcal{D}}_{i}$ be the prime divisors on $\mathcal{D}$ corresponding to the $\bar{D}_{i}$ for $1 \leq i \leq \beta$. Then we have

$$
j^{*} \mathcal{O}_{\mathcal{X}}\left(\mathcal{D}_{i}\right) \cong \mathcal{O}_{\mathcal{D}}\left(\overline{\mathcal{D}}_{i}\right)
$$

We note that $\left.D_{i}\right|_{D}=\frac{1}{t_{i}} \bar{D}_{i}$ in the usual language.

Theorem 6.1. (1) The functor $j_{*} \bar{\psi}^{*}: D^{b}(\operatorname{Coh}(\mathcal{F})) \rightarrow D^{b}(\operatorname{Coh}(\mathcal{X}))$ is fully faithful.

Let $D^{b}(\operatorname{Coh}(\mathcal{F}))_{k}$ denote the full subcategory of $D^{b}(\operatorname{Coh}(\mathcal{X}))$ defined by

$$
D^{b}(\operatorname{Coh}(\mathcal{F}))_{k}=j_{*} \bar{\psi}^{*} D^{b}(\operatorname{Coh}(\mathcal{F})) \otimes \mathcal{O}_{\mathcal{X}}\left(\sum_{i=1}^{n+1} k_{i} \mathcal{D}_{i}\right)
$$

for a sequence of integers $k=\left(k_{i}\right)$ for $1 \leq i \leq n+1$.

(2) If $0>\sum_{i=1}^{n+1} \frac{a_{i} k_{i}}{r_{i}} \geq-\sum_{i=1}^{n+1} \frac{a_{i}}{r_{i}}$, then

$$
\operatorname{Hom}^{q}\left(\Phi\left(D^{b}\left(\operatorname{Coh}\left(\mathcal{X}^{+}\right)\right)\right), D^{b}(\operatorname{Coh}(\mathcal{F}))_{k}\right)=0
$$

for all $q$.

(3) If $\sum_{i=1}^{n+1} \frac{a_{i} k_{i}}{r_{i}}>\sum_{i=1}^{n+1} \frac{a_{i} k_{i}^{\prime}}{r_{i}}>\sum_{i=1}^{n+1} \frac{a_{i}\left(k_{i}-1\right)}{r_{i}}$, then

$$
\operatorname{Hom}^{q}\left(D^{b}(\operatorname{Coh}(\mathcal{F}))_{k}, D^{b}(\operatorname{Coh}(\mathcal{F}))_{k^{\prime}}\right)=0
$$

for all $q$, where $k^{\prime}=\left(k_{i}^{\prime}\right)$ is another sequence of integers.

(4) If $\sum_{i=1}^{n+1} \frac{a_{i} k_{i}}{r_{i}}=\sum_{i=1}^{n+1} \frac{a_{i} k_{i}^{\prime}}{r_{i}}$, but if $j^{*} \mathcal{O}_{\mathcal{X}}\left(\sum_{i=1}^{n+1}\left(k_{i}-k_{i}^{\prime}\right) \mathcal{D}_{i}\right)=0$ or

$$
j^{*} \mathcal{O}_{\mathcal{X}}\left(\sum_{i=1}^{n+1}\left(k_{i}-k_{i}^{\prime}\right) \mathcal{D}_{i}\right) \notin \bar{\psi}^{*} D^{b}(\operatorname{Coh}(\mathcal{F}))
$$

then

$$
\operatorname{Hom}^{q}\left(D^{b}(\operatorname{Coh}(\mathcal{F}))_{k}, D^{b}(\operatorname{Coh}(\mathcal{F}))_{k^{\prime}}\right)=0
$$

for all $q$.

(5) The subcategories $\Phi\left(D^{b}\left(\operatorname{Coh}\left(\mathcal{X}^{+}\right)\right)\right)$and the $D^{b}(\operatorname{Coh}(\mathcal{F}))_{k}$ for

$$
0>\sum_{i=1}^{n+1} \frac{a_{i} k_{i}}{r_{i}} \geq-\sum_{i=1}^{n+1} \frac{a_{i}}{r_{i}}
$$

generate the triangulated category $D^{b}(\operatorname{Coh}(\mathcal{X}))$. 
Proof. (1) We shall prove that the natural homomorphism

$$
\operatorname{Hom}^{q}\left(L, L^{\prime}\right) \rightarrow \operatorname{Hom}^{q}\left(j_{*} \bar{\psi}^{*} L, j_{*} \bar{\psi}^{*} L^{\prime}\right)
$$

is bijective for all $q$ and all locally free sheaves $L$ and $L^{\prime}$ on $\mathcal{F}$.

We have an exact sequence

$$
\begin{aligned}
0 & \rightarrow \mathcal{O}_{\mathcal{X}}\left(-\sum_{i=\beta+1}^{n+1} \mathcal{D}_{i}\right) \rightarrow \cdots \rightarrow \bigwedge^{2}\left(\bigoplus_{i=\beta+1}^{n+1} \mathcal{O}_{\mathcal{X}}\left(-\mathcal{D}_{i}\right)\right) \\
& \rightarrow \bigoplus_{i=\beta+1}^{n+1} \mathcal{O}_{\mathcal{X}}\left(-\mathcal{D}_{i}\right) \rightarrow \mathcal{O}_{\mathcal{X}} \rightarrow j_{*} \mathcal{O}_{\mathcal{D}} \rightarrow 0
\end{aligned}
$$

Hence

$$
L_{q} j^{*} j_{*} \mathcal{O}_{\mathcal{D}} \cong \bigwedge^{q}\left(\bigoplus_{i=\beta+1}^{n+1} j^{*} \mathcal{O}_{\mathcal{X}}\left(-\mathcal{D}_{i}\right)\right)
$$

The sheaf $j^{*} \mathcal{O}_{\mathcal{X}}\left(-\sum_{i \in I} \mathcal{D}_{i}\right)$ for any subset $I \subset\{\beta+1, \ldots, n+1\}$ is either invertible or zero, and is negative for $\bar{\psi}$ if it is not a zero sheaf.

Since

$$
\omega_{\mathcal{D} / \mathcal{F}} \cong \mathcal{O}_{\mathcal{D}}\left(-\sum_{i=1}^{\alpha} \overline{\mathcal{D}}_{i}\right) \cong j^{*} \mathcal{O}_{\mathcal{X}}\left(-\sum_{i=1}^{\alpha} \mathcal{D}_{i}\right)
$$

we calculate

$$
\operatorname{Hom}^{q}\left(L_{p} j^{*} j_{*} \bar{\psi}^{*} L, \bar{\psi}^{*} L^{\prime}\right) \cong \operatorname{Hom}^{q}\left(\bar{\psi}^{*} L, \bar{\psi}^{*} L^{\prime} \otimes \bigwedge_{i=\beta+1}^{p}\left(\bigoplus_{i+1}^{n+1} j^{*} \mathcal{O}_{\mathcal{X}}\left(-\mathcal{D}_{i}\right)\right)\right) \cong 0
$$

for $p>0$ and for any $q$ by the relative vanishing theorem for $\bar{\psi}$, because $\sum_{i=1}^{n+1} \frac{a_{i}}{r_{i}}>0$. Hence

$$
\begin{aligned}
& \operatorname{Hom}^{q}\left(j_{*} \bar{\psi}^{*} L, j_{*} \bar{\psi}^{*} L^{\prime}\right) \cong \operatorname{Hom}^{q}\left(j^{*} j_{*} \bar{\psi}^{*} L, \bar{\psi}^{*} L^{\prime}\right) \\
& \cong \operatorname{Hom}^{q}\left(\bar{\psi}^{*} L, \bar{\psi}^{*} L^{\prime}\right) \cong \operatorname{Hom}^{q}\left(L, L^{\prime}\right)
\end{aligned}
$$

for any $q$ as required.

(2) It is sufficient to prove

$$
\operatorname{Hom}^{q}\left(\mathcal{O}_{\mathcal{X}}\left(\sum_{i=1}^{n+1} k_{i}^{\prime} \mathcal{D}_{i}\right), j_{*} \bar{\psi}^{*} A \otimes \mathcal{O}_{\mathcal{X}}\left(\sum_{i=1}^{n+1} k_{i} \mathcal{D}_{i}\right)\right)=0
$$


for all integers $q$, all sheaves $A$ on $\mathcal{F}$, and for the sequences $(k)$ and $\left(k^{\prime}\right)$ under the additional conditions that

$$
\begin{aligned}
& 0 \leq \sum_{i=1}^{n+1} \frac{a_{i} k_{i}^{\prime}}{r_{i}}<\sum_{i=\beta+1}^{n+1} \frac{b_{i}}{r_{i}} \\
& 0>\sum_{i=1}^{n+1} \frac{a_{i} k_{i}}{r_{i}} \geq-\sum_{i=1}^{n+1} \frac{a_{i}}{r_{i}} .
\end{aligned}
$$

It follows that

$$
0>\sum_{i=1}^{n+1} \frac{a_{i}\left(k_{i}-k_{i}^{\prime}\right)}{r_{i}}>-\sum_{i=1}^{\alpha} \frac{a_{i}}{r_{i}} .
$$

By the relative vanishing theorem for $\bar{\psi}$, we have

$$
\begin{aligned}
& \operatorname{Hom}^{q}\left(\mathcal{O}_{\mathcal{X}}\left(\sum_{i=1}^{n+1} k_{i}^{\prime} \mathcal{D}_{i}\right), j_{*} \bar{\psi}^{*} A \otimes \mathcal{O}_{\mathcal{X}}\left(\sum_{i=1}^{n+1} k_{i} \mathcal{D}_{i}\right)\right) \\
& \cong \operatorname{Hom}^{q}\left(j^{*} \mathcal{O}_{\mathcal{X}}\left(\sum_{i=1}^{n+1}\left(k_{i}^{\prime}-k_{i}\right) \mathcal{D}_{i}\right), \bar{\psi}^{*} A\right) \cong 0 .
\end{aligned}
$$

(3) is similarly proved as in (1). Since $0>\sum_{i=1}^{n+1} \frac{a_{i}\left(k_{i}^{\prime}-k_{i}\right)}{r_{i}}>-\sum_{i=1}^{\alpha} \frac{a_{i}}{r_{i}}+$ $\sum_{i=\beta+1}^{n+1} \frac{b_{i}}{r_{i}}$, we have

$$
R \bar{\psi}_{*} j^{*} \mathcal{O}_{\mathcal{X}}\left(\sum_{i \in I} \mathcal{D}_{i}+\sum_{i=1}^{n+1}\left(k_{i}^{\prime}-k_{i}\right) \mathcal{D}_{i}\right)=0
$$

for any subset $I \subset\{\beta+1, \ldots, n+1\}$ by the relative vanishing theorem for $\bar{\psi}$. Thus

$$
\operatorname{Hom}^{q}\left(j_{*} \bar{\psi}^{*} L, j_{*} \bar{\psi}^{*} L^{\prime} \otimes \mathcal{O}_{\mathcal{X}}\left(\sum_{i=1}^{n+1}\left(k_{i}^{\prime}-k_{i}\right) \mathcal{D}_{i}\right)\right)=0
$$

for all $q$ and all locally free sheaves $L$ and $L^{\prime}$ on $\mathcal{F}$.

(4) is similar to (3).

(5) We shall prove that the left orthogonal ${ }^{\perp} \mathcal{T}$ to the triangulated subcategory $\mathcal{T}$ of $D^{b}(\operatorname{Coh}(\mathcal{X}))$ generated by these subcategories consists of 0 objects.

Let $A$ be an arbitrary skyscraper sheaf of length 1 on $\mathcal{X}$ supported at a point $P$. If $P$ is not above a point in $D$, then $A \in \mathcal{T}$. Otherwise, there is 
a point $\bar{P}$ on $\mathcal{D}$ such that $P=j(\bar{P})$. Then by Theorem 3.6. there exists a skyscraper sheaf $B$ of length 1 on $\mathcal{F}$ supported at $\bar{Q}=\bar{\psi}(\bar{P})$ such that $A$ is contained in the subcategory generated by the sheaves of the form $j_{*} \bar{\psi}^{*} B \otimes$ $\mathcal{O}_{\mathcal{X}}\left(\sum_{i=1}^{n+1} k_{i} \mathcal{D}_{i}\right)$ for

$$
\sum_{i=\beta+1}^{n+1} \frac{b_{i}}{r_{i}}>\sum_{i=1}^{n+1} \frac{a_{i} k_{i}}{r_{i}} \geq-\sum_{i=1}^{n+1} \frac{a_{i}}{r_{i}}
$$

Therefore, $A$ contained in $\mathcal{T}$, hence ${ }^{\perp} \mathcal{T}=0$.

Corollary 6.2. Assume that $D^{b}\left(C o h\left(\mathcal{X}^{+}\right)\right)$has a complete exceptional collection consisting of sheaves. Then so has $D^{b}(\operatorname{Coh}(\mathcal{X}))$.

\section{References}

[1] A. A. Beilinson. Coherent sheaves on $\mathbb{P}^{n}$ and problems of linear algebra. Funct. Anal. Appl. 12(1978), 214-216.

[2] A. I. Bondal. Representations of associative algebras and coherent sheaves. Izv. Akad. Nauk SSSR Ser. Mat. 53 (1989), no. 1, 25-44; translation in Math. USSR-Izv. 34 (1990), no. 1, 23-42.

[3] A. I. Bondal and M. M. Kapranov. Representable functors, Serre functors, and reconstructions. Izv. Akad. Nauk SSSR Ser. Mat. 53 (1989), no. 6, 1183-1205, 1337; translation in Math. USSR-Izv. 35 (1990), no. 3, 519-541.

[4] A. I. Bondal and D. O. Orlov. Reconstruction of a variety from the derived category and groups of autoequivalences. alg-geom/9712029. Compositio Math. 125 (2001), 327-344.

[5] Tom Bridgeland. Equivalences of triangulated categories and Fourier-Mukai transforms. math.AG/9809114. Bull. London Math. Soc. 31(1999), 25-34.

[6] Tom Bridgeland. T-structures on some local Calabi-Yau varieties. math.AG/0502050. 
[7] A. L. Gorodentsev, S. A. Kuleshov and A. N. Rudakov. tstabilities and t-structures on triangulated categories. (Russian) Izv. Ross. Akad. Nauk Ser. Mat. 68 (2004), no. 4, 117-150. math.AG/0312442.

[8] M. M. Kapranov. Derived category of coherent sheaves on Grassmann manifolds. Izv. Akad. Nauk SSSR Ser. Mat. 48 (1984), no. 1, 192-202.

[9] M. M. Kapranov. On the derived category and $K$-functor of coherent sheaves on intersections of quadrics. Izv. Akad. Nauk SSSR Ser. Mat. 52 (1988), no. 1, 186-199; translation in Math. USSRIzv. 32 (1989), no. 1, 191-204.

[10] M. M. Kapranov. On the derived categories of coherent sheaves on some homogeneous spaces. Invent. Math. 92 (1988), no. 3, 479508.

[11] Yujiro Kawamata. Euivalences of derived catgories of sheaves on smooth stacks. math.AG/0210439. Amer. J. Math. 126(2004), 1057-1083.

[12] Yujiro Kawamata. Log crepant birational maps and derived categories. math.AG/0311139

[13] Yujiro Kawamata, Katsumi Matsuda and Kenji Matsuki. Introduction to the minimal model problem. in Algebraic Geometry Sendai 1985, Advanced Studies in Pure Math. 10 (1987), Kinokuniya and North-Holland, 283-360.

[14] Emanuele Macri. Some examples of moduli spaces of stability conditions on derived categories. math.AG/0411613.

[15] Kenji Matsuki. Introduction to Mori Program. Springer, 2002.

[16] D. O. Orlov. Projective bundles, monoidal transformations, and derived categories of coherent sheaves. Russian Acad. Sci. Izv. Math. 41(1993), 133-141.

[17] Miles Reid. Decomposition of toric morphisms. Arithmetic and geometry, Vol. II, 395-418, Progr. Math., 36, Birkhauser Boston, Boston, MA, 1983. 
[18] Alexei N. Rudakov. Rigid and exceptional vector bundles and sheaves on a Fano variety. Proceedings of the International Congress of Mathematicians, Vol. 1, 2 (Zurich, 1994), 697-705, Birkhauser, Basel, 1995.

Department of Mathematical Sciences, University of Tokyo, Komaba, Meguro, Tokyo, 153-8914, Japan

kawamata@ms.u-tokyo.ac.jp 